
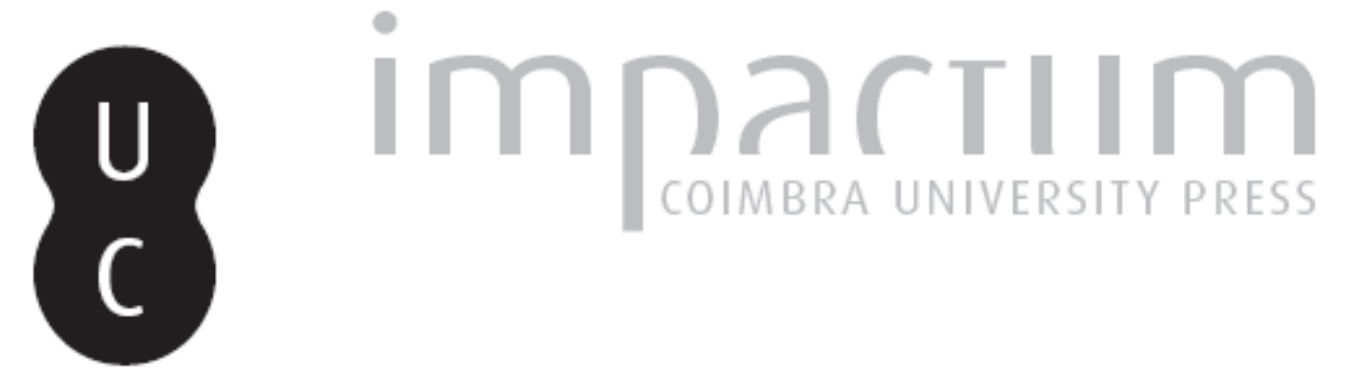

\title{
Una lectura 'lógica' de Phaid: 100a3-7
}

Autor(es): Mombello, Eduardo Héctor

Publicado por: Imprensa da Universidade de Coimbra

URL persistente:

URl:http://hdl.handle.net/10316.2/42285

DOI:

DOl:https://doi.org/10.14195/2183-4105_3_1

Accessed : $\quad$ 26-Apr-2023 06:07:39

A navegação consulta e descarregamento dos títulos inseridos nas Bibliotecas Digitais UC Digitalis, UC Pombalina e UC Impactum, pressupõem a aceitação plena e sem reservas dos Termos e Condições de Uso destas Bibliotecas Digitais, disponíveis em https://digitalis.uc.pt/pt-pt/termos.

Conforme exposto nos referidos Termos e Condições de Uso, o descarregamento de títulos de acesso restrito requer uma licença válida de autorização devendo o utilizador aceder ao(s) documento(s) a partir de um endereço de IP da instituição detentora da supramencionada licença.

Ao utilizador é apenas permitido o descarregamento para uso pessoal, pelo que o emprego do(s) título(s) descarregado(s) para outro fim, designadamente comercial, carece de autorização do respetivo autor ou editor da obra.

Na medida em que todas as obras da UC Digitalis se encontram protegidas pelo Código do Direito de Autor e Direitos Conexos e demais legislação aplicável, toda a cópia, parcial ou total, deste documento, nos casos em que é legalmente admitida, deverá conter ou fazer-se acompanhar por este aviso. 


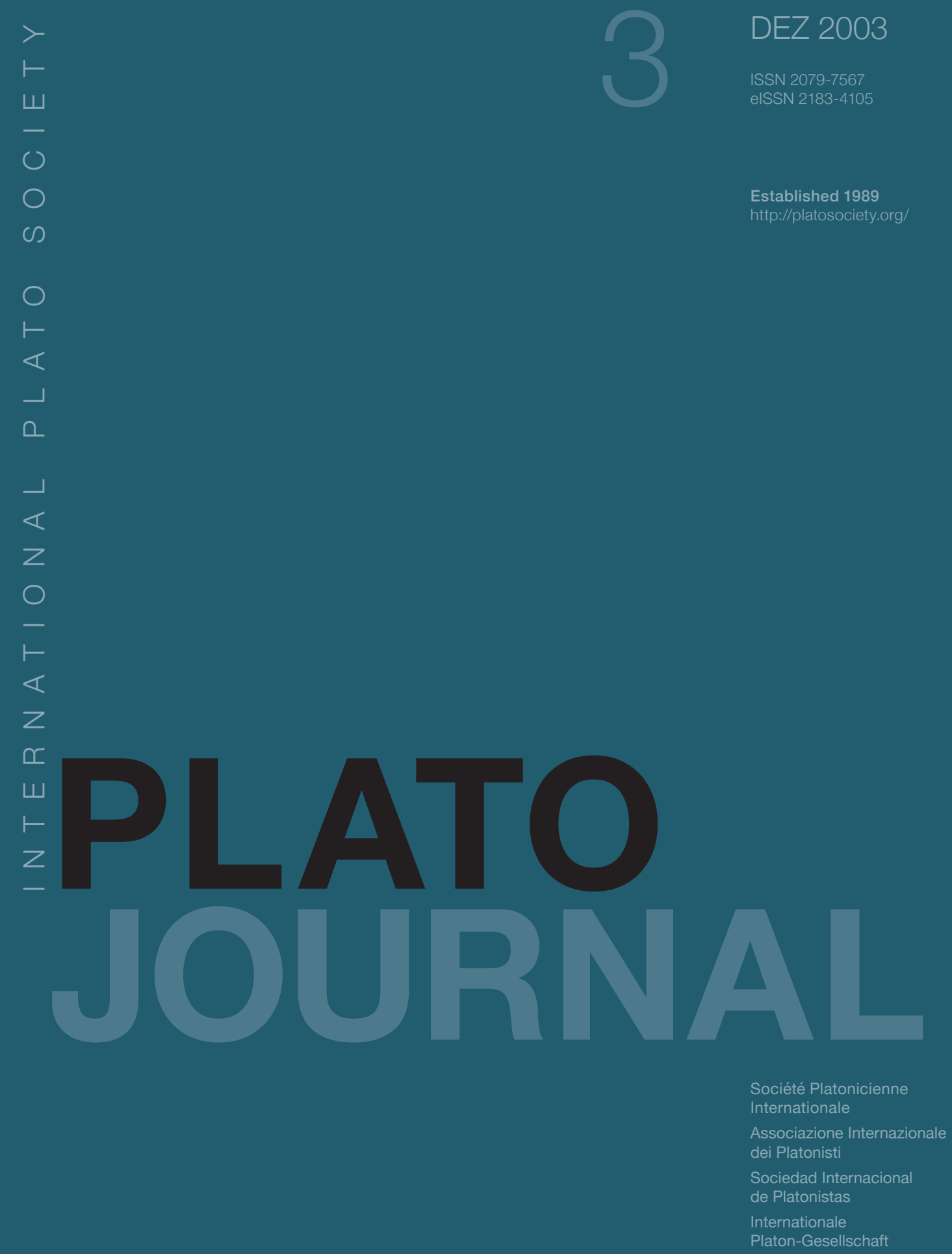




\title{
Una lectura 'lógica' de Phaid. 100a3-7
}

\author{
MOMBELLO, Eduardo Héctor, in 3. Plato
}

3 (2003) , [En ligne] , January 2003

Platón ha presentado una táctica tendiente a encuadrar una investigación en la actividad que consiste en discutir y descubrir algo de lo que es (ti tôn ontôn - Fedón101e3), que desde 99c9-d se ha llamado deuteros plous. Ella invita a determinar esquemáticamente las relaciones que, desde su perspectiva ontológica y sobre la base de ciertas aitiai, se establecen con lo que es. Así, si hay que conducirse como filósofos (101e6), habrá que seguir el procedimiento propuesto para la consideración de las cosas que son (ta onta 99d5): estudiar los enunciados (logoi) para reconocer en ellos «la verdad de las cosas que son» (99e4-6). Una variedad importante de explicaciones y ejemplos de esa táctica (99e4-107b10) han intentado ser esquematizados lógica y coherentemente por la crítica con resultados variables, en ocasiones, desalentadores [1].

Mi trabajo se concentra sólo en la noción central de (100a5; 101d5) que Platón usa allí. De este modo, defiendo la pertinencia del uso de un recurso 'lógico' simple, según surge del análisis de esa noción que resulta crucial para la comprensión de su táctica. Precisamente, sostengo que $(t)$ tanto el criterio de determinación del valor de verdad de enunciados 'concordantes' expresado en 100a4-7, como la noción de 'sumphônein' deben ser comprendidos, desde su aspecto lógico, como nuestra noción de 'entrañamiento'. Pero $(t)$ forma parte de un estudio más amplio sobre la relación entre lógica y ontología presente en la expresión de la llamada 'teoría de la ideas' de Fedón100b-102a2. La tesis general que lo guía - y que en este trabajo no supera el estado de supuesto- es que el recurso a la lógica estándar (de primer orden, extensional y cuantificacional) resulta insuficiente para el análisis de las relaciones lógicas subyacentes a aquella exposición de la doctrina de las ideas, por varias razones. Sobre el final, al considerar el alcance de $(t)$, discutiré la que creo señala mejor el problema: que tal recurso carece de los elementos pertinentes, i.e., de los signos y de la respectiva gramática adecuada a su ontología [2]: el 'marco ontológico' ( $c f$. infra c.2) requerido por la herramienta que solemos utilizar es en todo diverso del propuesto por Platón. Sobre esa base, intento mostrar, de modo prospectivo, una interpretación de cierta parte de los lineamientos básicos y comunicables de su ontología que recurre a la adecuación de un instrumental 'lógico' ad hoc.

Uno de los análisis más influyentes de la noción de sumphônein es el agudo trabajo de Richard Robinson (1953). Su estudio ilustra, sin embargo, parte de las limitaciones que el recurso a la axiomatización estándar [3] parece tener para capturar las nociones 'lógicas' de Platón como la de sumphônein. En efecto, su enfoque sobre el valor de la noción en 100a, es un enfoque formal. Desde su perspectiva, sumphônein no puede aludir más que a (1) 'ser consistente con' o a (2) 'ser deducible de' (o bien a 'ser implicado por'), queriendo señalar con ello que la proposición es una consecuencia lógicamente necesaria de la hupothesis (p.126). 'No hay una tercera interpretación' (p.127). Por ello, su propia exégesis termina por asumir que Platón no dice todo lo que quiere decir, lo cual - a su juicio - se sintetiza en la combinación de ambos predicados: «Él sólo dice que el segundo paso del método es encontrar proposiciones consistentes con la hipótesis, pero quiere decir que se encuentren proposiciones no meramente consistentes con la hupothesis sino también deductibles de ella» (p.128). Esta conjunción sería el significado de sumphônein no explicitado por Platón, sino aludido metafóricamente. En virtud de esto y del paso 2 [4] del método general que atribuye a Platón, Robinson compromete su explicación de la propuesta metodológica - al menos - con el siguiente enunciado: si Platón postula a $p$ como verdadera y a $\emptyset p$ como falsa, entonces la proposición $p$ se sigue de (o es implicada por) la hipótesis $H$. Pero razones lógicas muestran que ese enunciado es verdadero aun cuando $p$ no se siga de $H$, i.e., aun cuando los enunciados no sean 'concordantes'. Su explicación no sólo dice más de lo que dice Platón, sino que podría decir, además, algo que él no estaría dispuesto a asumir: que aun cuando $p$ sumphônein con la hipótesis $H$, Platón podría no aceptar a $p$ como verdadero y a $\emptyset p$ como falso.

La posición de Robinson parte del supuesto de que, si el significado de sumphônein no se identifica con la noción de consecuencia lógica, entonces $p$ sumphônei con $H$ se asimila al de una fórmula bien formada del lenguaje que excluye a la de la contradicción. Omite el caso de que sumphônein no sea una noción perteneciente al metalenguaje que estudia al lenguaje formal objeto, o una noción expresable en este último. Creo que no hay necesidad de suponer tal cosa. He utilizado para el título de este trabajo la palabra 'lógica' así, entre comillas, justamente, porque si bien haré uso de esa herramienta para descubrir parte de lo que Platón ha podido 'querer decir' consumphônein, no identifico su noción con ninguna pieza conceptual de la herramienta de la cual hago uso, puesto que, como se verá, eso no parece aconsejable. Dicho de otro modo, si conviene a nuestro estudio analizar a Platón en términos de una lógica formal desarrollada 'fuera' de sus teorías, lo es, justamente, no porque vayamos a identificar sus nociones con las nuestras, sino porque la limitación de nuestra herramienta descubre, en este caso, el tamaño de la suya. Así, el enfoque de Robinson parece haber omitido una cuestión central: la relación de sumphônein está enmarcada en un criterio de verdad para los enunciados 
involucrados en ella que es lógicamente anterior (por necesario) al 'significado lógico' o 'metalógico' que podamos encontrar para el signo «sumphônein». Criterio propio que sí puede ser formalizado a partir del usoplatónico que explica la relación sumphônein con que vincula los logoi mencionados, aun desconociendo lo que, por ella, debemos comprender. Esto, precisamente, marca los límites que acotan el significado buscado según se lo puede esclarecer, posterior y parcialmente, desde una perspectiva 'lógica'. Ésta debe, cuanto menos, poder adaptarse al nudo de coincidencia temática de análisis por el 'refugio en los enunciados' que propone Platón desde Phaid. 99d4ss []], cuya relación central se capta - a mi juicio - en $(t)$.

El modo de proceder que Platón propone presenta dos momentos gravitantes:

国 aquel en que se toma como base (hupothemenos) el enunciado (logos) que se juzga ser más sólido (errômenestatos, 100a3-4). 国. aquel que establece que se toman como cosas que son verdaderas (alêthê onta) lo que parece consonar (sumphônein) con el enunciado establecido según el criterio de (a). Esto vale tanto para los logoi que expresan las aitiai, como para aquellos que lo hacen sobre las demás cosas [que son] (100a4-6). Lo que no concuerde con los enunciados obtenidos según el momento $(a)$ será tenido por no-verdad (100a7).

Aunque no es el tema del presente, el peso que el momento $(a)$ tiene sobre el $(b)$ amerita una breve reflexión. En general, debe tenerse presente que $(a)$ es condición de posibilidad de $(b)$. De lo que se sigue la imposibilidad de acceso a cosas que son verdaderas si no se cuenta con las exigencias del primer momento. En particular, qué significa allí errômenestatos (literalmente, fortísimo) es una cuestión que no debe pasarse por alto. En efecto, aunque en el pasaje Platón no aclara el punto, podríamos tener en mente ciertos candidatos. Que un logos tal es aquel:

a.1 - capaz de generar un firme convicción o una rápida adhesión. Es decir, es aquello capaz de ser contenido proposicional de un enunciado no extensional del tipo «creo que $x$ » donde $x$ es una variable proposicional.

(10 a.2 - en virtud del cual cobran sentido otros enunciados (lo que le otorga cierto valor heurístico) en tanto es un principio (archê 101e1-3). Estos enunciados derivados tendrían la peculiar característica de reforzar la cuota de crédito otorgado al sólido logos. Tal anuencia se ampliaría en la medida en que se logra una explicación adecuada de determinados eventos o fenómenos de diversa índole. En este sentido, la noción de enunciado errômenestatos sería contraria a la de eikôs logos [ 6 ] (un enunciado verosímil o apenas probable).

a.3 - que afirma la existencia de cosas que no tienen un carácter sensoperceptible. La fuerza de tal enunciado radicaría en su capacidad para el establecimiento de relaciones entre el plano del lenguaje y cosas no evidentes (sensoperceptibles). En términos actuales, es un enunciado que fija su candidato a referente por la vía de las condiciones que éste debe cumplir. Tales condiciones no son más que los predicados básicos por los que a tal referente se significa sin nombrarlo por el recurso corriente del uso de un término singular o expresión deíctica.

El ejemplo que ilustra este momento (a) muestra al personaje Sócrates que toma como base (hupothemenos) que (e1) «existe algo bello en sí y por sí (ti kalon auto kath 'hauto) y a.1 del logos que se da como ejemplo se evidencia en la dispuesta concesión de Cebes (100c), el interlocutor de Sócrates a estas alturas. Más ajustado aún, por cumplir además con el aspecto $a .3$, es el caso de la adhesión de Simias en 77a2-5:

Ciertamente, yo por mi parte nada tengo por más evidente (enarges) que esto: que todas estas cosas existen en grado superlativo (malista), tanto lo bello y bueno como todas las otras cosas que acabas de decir.

El caso se repite nuevamente por la indubitable y vehemente adhesión de Simias a la afirmación de la existencia de lo igual en sí (auto to ison) en 74b. No hay que pasar por alto que los tres pasajes (74b; 77a2-5; 100c) mostrados aquí como ejemplos de aparición del carácter $a .1$, muestran además la coincidencia del carácter $a .3$ respecto de aquello a lo que la adhesión y convicción se aplican. Todos revelan, así, el estado de creencia del personaje sobre un juicio de existencia de objetos no-sensoperceptibles. Pero esto no debe confundirse con una afirmación de existencia en sentido fuerte de tal tipo de entidades, pues, por el carácter $a .1$ que estamos analizando, tales afirmaciones no son más que contenido proposicional de un juicio intencional. Estamos hablando de Platón, no de Meinong. Los juicios intencionales parecen coronar siempre contextos de demostración o explicación de estados de cosas que involucran 'entidades posibles', es decir, no sensoperceptibles. Tal tipo de contextos recorren prácticamente todo Fedón en busca de la demostración de la inmortalidad del alma. El enunciado tomado aquí como fundamento (hupothemenos) - que en $101 \mathrm{~d} 2$ ss. es significado por «hupothesis» - tiene, no cabe duda, el carácter $a$.1. En efecto, como todas las afirmaciones de existencia involucradas en estos ejemplos, se trata de unahupothesis «digna de darse a conocer». El pasaje (92c11-e3) en que Simias da las razones por las cuales considera que el aprendizaje es reminiscencia tampoco deja lugar a dudas y, además, se expone allí que, de los logoi que no se demuestran a partir de una hupothesis digna de darse a conocer [7] - sino «con la ayuda de algo meta eikotos tinos -, no pueden esperarse más que engañosas charlatanerías. El caso es que en todos los pasajes que aquí se presentaron, la afirmación fuerte se ofrece en vista de una explicación ulterior (carácter a.2) y consiste siempre en la postulación de estados de cosas no evidentes por involucrar la existencia de objetos no-sensoperceptibles (carácter $a .3$ ), sean estos el alma o su inmortalidad, o cualquiera de las cosas a las que sellamos con «auto ho esti» [8].

\section{b.1 Un criterio veritativo para los enunciados concordantes.}

El segundo momento señalado ( $c f$. b supra) nos dispone, al fin, en busca de la «verdad de las cosas que son» (99e5-6). La idea es que 
los enunciados que concuerden o ‘consuenen' con el enunciado que se tome como base, serán verdaderos. La alegoría sinfónica parece hablar de alusiones diferentes - por ser diversos los instrumentos, loslogoi - a una misma melodía; a un mismo referente o estado de cosas dentro de un única argumentación.

Con vista a una formalización de lo dicho en esas líneas, podemos tomar $r$ como el contenido proposicional [9] del enunciado errômenestatos y $s$ como el contenido proposicional del logos candidato. Un problema, no menor, se presenta con «sumphônei». En efecto, es indudable que con esa relación Platón no hace uso sino mención del lenguaje: vincula con ella dos proposiciones. Por ello, tendríamos que ser capaces de expresar que 's sumphônei con $r$ ', más allá de lo que «sumphônei con» signifique en otros usos, pues, al menos lógicamente (y lo que aquí interesa es efectivamente ver cómo se vincula a los valores de verdad de sus argumentos), no lo sabemos. Lo que sí sabemos es que en otros diálogos Platón hace un uso semánticamente variado del términ [10], aunque no parece difícil encontrar algunas notas comunes a todos ellos relacionada a la noción de semejanza ( $c f$. República 617b7; 402d1-3; Cratilo [11] 415b2 [12]; 402c2; 433b4; Teeteto 154d8-e5). Explícitamente, leemos en Timeo 80a5-6 que los sonidos pueden presentársenos como consonantes en virtud de la semejanza. Esa nota también prevalece en el sentido lógico que intentamos delimitar parasumphônein: los valores de verdad de los enunciados involucrados en una explicación causal así relacionada, son trivialmente semejantes. Sin ánimo de introducir más elementos al cálculo lógico que los necesarios, pero con el interés de ver el alcance 'lógico' de lo que Platón propone, podríamos simbolizar 'sumphônei con' con ' $\Sigma$ ' y tomarla provisoriamente como si fuéramos a transformarla en un símbolo lógico por definir que relacione proposiciones.

Ahora bien, si s sumphônei con $r$, entonces $s$ es verdadero. Esto significa que la verdad de $s$ se sigue necesariamente de la verdad de $\Sigma r s$ (lo que hay que leer «s sumphôneicon $r »)$. Formalmente (1): $\Sigma r s \circledR s$. Contrariamente, si no es cierto que $s$ sumphônei con $r$, entonces no será cierto que $s$ es verdadero. Y esto significa que la falsedad de $s$ se sigue necesariamente de la falsedad de $\Sigma r s$; o sea (2) Ø $\Sigma r s ® \emptyset$. Forzosamente, entonces, s sumphônei con $r$, si, y sólo si, $s$ es verdadero. E inversamente, s nosumphônei con $r$, si, y sólo si, $s$ no es verdadero; es decir, $\Sigma r s \ll s$ y $\varnothing r s \ll \emptyset s$ son restricciones que se siguen de la relación propuesta por Platón en la que, desde el comienzo, hemos concedido - al menos provisoriamente - la verdad de $r$.

Esquemáticamente, por (1) y (2) el criterio veritativo para los enunciados derivados se expresa:

\section{(3) $\left(\sum r s{ }^{\circledR} s\right) \grave{U}(\emptyset \Sigma r s \circledR \emptyset s)$}

Lo que esto dice es que la verdad de $s$ se sigue necesariamente de que $s$ sumphônei con $r$; y que, si esto no ocurre, se sigue necesariamente la no-verdad de $s$. Sugiero que, en vistas a elucidar lo que el término «sumphônein» involucra, el criterio veritativo debe ser entendido como una relación de entrañamiento. En efecto, esta relación entre proposiciones - desde el plano estrictamente formal - se establece como: dadas dos proposiciones $q$ y $s, q$ entraña $s(q P s)$ [13] si, y sólo si, la verdad de $s$ se sigue necesariamente de la verdad de $q$; inversamente, la falsedad de $s$ debe inferirse necesariamente de la falsedad de $q[\underline{14}]$. Podemos expresar la equivalencia lógica del entrañamiento de $q$ a $s$, como

$(q P s) \ll[(q ® s) \grave{\mathrm{U}}(\emptyset q \circledR \varnothing s)]$

y esto es lo que lógicamente significa, en lo que toca al criterio veritativo, la propuesta esquematizada (3) de Platón. La forma abreviada, entonces, del criterio veritativo podría parafrasearse como sigue:

$\sum r s P$.

Es claro que Platón no llama a este momento $(b)$ del análisis de los logoi «criterio veritativo» tanto como que en esas mismas líneas (100a4-7) se expresan dos relaciones fundamentales entre cosas no bien explícitas y que se han tomado aquí como proposiciones o contenido proposicional de los enunciados (logoi) [15]. Una es la llamada explícitamente «symphōnein», y de la que no se nos dice en qué consiste; la otra, que no es nombrada pero cuyas características básicas son definidas por Platón, es la que hemos llamado aquí criterio veritativo para $s$. Este criterio veritativo para $s$ consiste, entonces, en la relación de entrañamiento entre una proposición que expresa la consonancia de la proposición $s$ con la proposición $r$, y la proposición $s$ - que consuena con $r$-. Queda por ver, sin embargo, qué significa ' $\Sigma r s$ ' lógicamente.

\section{b.2 Una relación como garantía para el criterio veritativo}

El hecho de que $\Sigma r s$ entrañe $s$, establece dos restricciones. Por un lado, indica que no puede ocurrir que $s$ sea falso si $r$ sumphônei con $s[\emptyset(\Sigma r s \circledR \emptyset s)]$; por el otro, que es imposible que ocurra que no sumphônei $r$ con $s$ y $s$ sea verdadera $[\emptyset(\emptyset \Sigma r s \circledR$ s)]. Es decir,

$(\operatorname{\Sigma rs} P s) \circledast[\varnothing(\operatorname{\Sigma rs} 囚 \emptyset)]$

$(\Sigma \mathrm{rsP} s) \circledast[\emptyset(\varnothing \Sigma r s \circledR s)]$.

Atento a esto, podemos proseguir la investigación del significado lógico de ' $\Sigma r s$ '. Tomando las restricciones recién vistas, y los dos casos dados por Platón, en que $r$ es siempre verdadero y $s$ cambia su valor de verdad según consuene o no con $r$, podemos buscar la definición lógica de $\Sigma$ en una tabla de verdad tal que contemple lo que se ha visto hasta ahora para el criterio veritativo ( $c f$. el 
cuadro infra) y el comportamiento de $r, s$ y $\Sigma$, según los valores que puede asumir el criterio (3).

Las líneas ii y iv del cuadro reflejan lo que el significado, que hemos asumido de lo expresado por Platón, establece para el 'criterio veritativo' (3):

b.2.1 que si ss sumphônei con $r$, entonces $s$ es verdadero (línea ii de la tabla).

b.2.2 que si no es cierto que $s$ sumphônei con $r$, entonces $s$ es falso (línea iv). Esta es la restricción que Platón establece en 100 a7. b.2.3 en ambos casos (b.2.1 - b.2.2) conocemos el valor de verdad de $r$, porque lo hemos concedido según $a .1$, esto es, que $r$ es verdadera.

Esto es todo lo que Platón nos dice. Por otra parte, las líneas i y iii corresponden a las restricciones que se establecieron por el criterio veritativo. En esas mismas líneas, entonces, en la parte correspondiente a la definición de ' $\Sigma \Sigma$ ’? conocemos los estados veritativos que toman $\Sigma$ y $s$ a partir del cuadro del criterio (3). Ahora bien, el valor de verdad del enunciado errômenestatos $r$ en la línea i, podría ser tanto 'v' como ' $\mathrm{f}$ '. Pero, si admitimos que el enunciado errômenestatos $r$ es verdadero, y es verdad que sconsuena con éste (lo cual está establecido en la misma línea del cuadro de (3), al igual que el valor para $s$ en esa línea), estaremos contradiciendo lo que propone Platón para el caso ii. Estaríamos admitiendo la posibilidad de que aún cuando $r$ sea verdadero y $s$ consuene con él, $s$ pueda ser falso. Luego, no queda más que aceptar que, para la línea i, $r$ debe ser falso. Una simple inspección del cuadro muestra que una situación similar se presenta en la línea iii, y de modo semejante también se soluciona, por lo cual no nos detendremos en ello.

\begin{tabular}{|c|c|c|c|c|c|c|c|c|c|c|c|c|c|c|}
\hline \multicolumn{4}{|c|}{ Definición de $\Sigma$} & \multicolumn{11}{|c|}{ Criterio veritativo (3) para $s$} \\
\hline \multicolumn{2}{|l|}{$r$} & \multirow{2}{*}{$\begin{array}{l}\Sigma \\
\mathbf{v}\end{array}$} & \multirow{2}{*}{\begin{tabular}{|l|}
$s$ \\
$\mathbf{f}$ \\
\end{tabular}} & \multirow{2}{*}{\begin{tabular}{|l}
$r s$ \\
$\mathrm{~V}$
\end{tabular}} & \multirow{2}{*}{$\begin{array}{l}\circledR \\
\mathbf{f}\end{array}$} & \multirow{2}{*}{$\begin{array}{l}s \\
\mathrm{f}\end{array}$} & \multirow{2}{*}{\begin{tabular}{|l}
$\grave{U}$ \\
$\mathbf{f}$
\end{tabular}} & \multirow{2}{*}{\begin{tabular}{|l}
$\varnothing \Sigma r s$ \\
$\mathrm{f}$
\end{tabular}} & \multirow{2}{*}{\begin{tabular}{|l} 
(1) \\
$\mathrm{V}$
\end{tabular}} & \multirow{2}{*}{\begin{tabular}{|l}
$\varnothing_{s}$ \\
$\mathrm{v}$
\end{tabular}} & \multicolumn{2}{|c|}{$\Sigma r s$} & \multirow{2}{*}{$\begin{array}{l}P \\
\mathrm{f}\end{array}$} & \multirow{2}{*}{$\begin{array}{l}s \\
\mathrm{f}\end{array}$} \\
\hline $\mathrm{i}$ & $\mathbf{f}$ & & & & & & & & & & (iv & $\mathrm{V}$ & & \\
\hline ii & $\mathbf{v}$ & $\mathbf{v}$ & $\mathbf{v}$ & $\mathrm{V}$ & $\mathrm{v}$ & $\mathrm{V}$ & $\mathrm{v}$ & $\mathrm{f}$ & $\mathrm{v}$ & $\mathrm{f}$ & (ii) & $\mathbf{v}$ & $\mathbf{v}$ & $\mathbf{v}$ \\
\hline iii & $\mathrm{f}$ & $\mathbf{f}$ & $\mathrm{v}$ & $\mathrm{f}$ & $\mathrm{v}$ & $\mathrm{v}$ & $\mathbf{f}$ & $\mathrm{v}$ & $\mathbf{f}$ & $\mathrm{f}$ & (iii & f & $\mathrm{f}$ & $\mathrm{V}$ \\
\hline iv & $\mathrm{v}$ & $\mathbf{f}$ & $\mathrm{f}$ & $\mathrm{f}$ & $\mathrm{v}$ & $\mathrm{f}$ & $\mathrm{v}$ & $\mathrm{v}$ & $\mathrm{v}$ & $\mathrm{V}$ & (i) & $\mathbf{f}$ & $\mathbf{v}$ & $\mathbf{f}$ \\
\hline
\end{tabular}

Qué puede ocurrir en el caso de consonancia i o de no consonancia iii siendo $r$ falso, es algo que a Platón no le interesa. Aunque podría argüirse en favor de que tal desinterés se basa, en ambos casos, en que en esas situaciones no se estaría hablando de nada y, consecuentemente, nada explicarían. Esto refuerza la importancia del aspecto a.3 visto arriba.

Finalmente, es evidente - por definición de $\Sigma$-, que cuando s sumphônei con $r$ (casos i y ii), si $r$ es verdadera, $s$ es necesariamente verdadera (ii) y si $r$ es falsa, $s$ es necesariamente falsa (i); lo cual muestra que, desde un punto de vista lógico (dado el criterio establecido por Platón en 100a5-7) y tal cual se ve en la definición de entrañamiento a la derecha del cuadro, «sumphônein» puede ser entendido, en este acotado sentido - siendo aquella relación condición suficiente para ello -, por «entrañar». Pero no hay que olvidar que esto sólo es un aspecto muy limitado de tal noción, y que Platón sólo establece lo que se sigue de las líneas ii y iv. Las otras dos líneas estarían, para Platón, fuera del método propuesto, por no darse el caso de que $r$ sea verdadera, aunque, como hemos visto, se siguen lógicamente. Entonces la relación entre $r$ y $s$ es - al menos - tal que

\section{$\operatorname{\sum rs} ®(r P s) .[\underline{16}]$}

Aunque no es cuantiosa la información que esto nos aporta para el análisis de las explicaciones causales en las que está enmarcado el método de estudio de las cosas que son en los logoi, sí podemos determinar - y esto es lo relevante - que si no se da el caso de que $(r P s)$, tampoco el de que $\Sigma r s$. Lo mismo vale para los enunciados que completan la explicación causal. Ciertamente, los predicados por los que se determinan las entidades cuya existencia se afirma en la hupothesis $r$, deben permitir la relación de entrañamiento recíproco ( $c f .101 \mathrm{~d} 3-5)$ entre los enunciados $(s, s, s$ ", ...) involucrados en la explicación posterior. Y tal cosa significa que estos enunciados están dando cuenta, o reflejando, un mismo estado de cosas y, en tal sentido, las proposiciones involucradas pueden ser tomadas como 'cosas que son verdad'.

Como es claro, el valor veritativo de $s$ - y de todo enunciado así obtenido - depende necesariamente del de $r$, de allí la importancia de lo observado en $a .1$. No se puede evitar, entonces, que en esta última afirmación el problema recaiga sobre la noción de necesidad. Parece plausible poder argüir que - al menos hasta este punto - sólo se está hablando de lo que se entiende por necesidad lógica [17].

\section{c. Alcance y ámbito de aplicación de (t).}

En la ejemplificación del segundo momento, se requiere la admisión del siguiente enunciado causal: (e2) «si hay alguna otra cosa bella además de lo bello en sí, no es bella por ningún otro motivo que el de que participa de aquél lo bello» 〈¿en sí?> (100c5-6). Si esto es un perfecto ejemplo de consonancia de un enunciado causal con su logoserrômenestatos, podremos ver que, si (e1) es verdadero, la proposición causal (e2) debe ser necesariamente verdadera. En efecto, si hay una clave que abra la posibilidad de dar 
cuenta de esto sin entrar en enredadas explicaciones que suponen nociones lógicas ajenas al marco ontológico en cuestión [18], ella está en el tipo de predicados por el que se determina lo que en otros contextos se menta con «idea». Lo cual se debería revelar por el estudio detenido de los contenidos proposicionales (e1) y (e2) que se ofrecen como ejemplos en estos dos momentos de la explicación. ¿Son ‘lo-bello’ o ‘lo-bello-en-sí’ nombres propios de las ideas? ¿Hacemos bien a la claridad del pensamiento platónico al traducir con mayúsculas 'lo Bello'? Creo que hay suficiente evidencia de que las respuestas podrían ser negativas: antes de analizar cómo (e2)sumphônei con (e1) - sobre lo que amerita volver - se hace necesario el análisis de algunas cuestiones previas. Dado el carácter provisorio - cf. supra, especialmentea.1 a.3 - de los juicios de existencia de las entidades involucradas en las 'ideas', y las fórmulas de expresión preferidas por Platón para su determinación, mucho se gana (al menos en claridad y consistencia) en la lectura de la ontología platónica, si se toman los nombres de las ideas no como 'nombres propios' de cada una de ellas, sino como descripciones definidas, según las han entendido Russell [19] primero, y Quine [20] después. Pero esto y la aplicación de sumphônein en su ámbito requieren, para dar cuenta de los tipos de explicaciones expuestas por Platón, precisar algunos puntos sobre el alcance y limitaciones de nuestra propuesta, de modo prospectivo:

(1) Lo que esto no dice es que debamos comprender la hupothesis (que supone cuantificación) como una descripción definida, sino a las expresiones nominales de las que Platón hace uso cuando alude a las ideas. Esto es «lo-Bello-en-sí», «lo-Bueno-en-sí», et cetera.

(1.1) El enfoque de Platón para este método no se cambia aceptando (1). Ciertamente, de ese modo el compromiso ontológico no recae en los mismos términos utilizados para describir tales entidades, sino en el verbo por el que la proposición errômenestatos afirma la existencia de las mismas. Y esto es coherente con el tratamiento de las descripciones pues, como se sabe, ellas no nombran nada, es decir, no aseveran la existencia de nada, lo cual hace necesaria su explicitación en los contextos demostrativos que así lo requieran [21]. Según (a.), las proposiciones (hupotheseis) en las que se introducen las ideas son juicios de existencia que requieren siempre la concesión del interlocutor.

(2) Voy a llamar 'marco ontológico' [22] a cualquier concepción aceptada sobre lo que hay/existe, a la jerarquía de los diferentes tipos de objetos (primarios y abstractos, por ejemplo), a sus relaciones, etc.; cosas que damos por supuestas en todo análisis y que nos permiten diferenciar, en el lenguaje, entre los individuos y las propiedades que ellos pueden tener. Sólo suponiendo esta diferencia para un marco ontológico unívoco dado podríamos contar con proposiciones susceptibles de ser simbolizadas (o esquematizadas) y de tener un valor de verdad por referencia a él ( $c f$. n.2). El lenguaje del marco manifiesta el compromiso de la jerarquía entre sus objetos primarios y no-primarios, pero no necesariamente el de la existencia de los mismos. Y voy a usar la palabra 'nuestro' ('nosotros', etc.) para aludir al marco ontológico en el que larealidad primaria está constituida por individuos sensoperceptibles (familiares) y los restantes son abstractos, o clases, o nociones, o predicados, o inexistentes, etc. Al menos, las relaciones entre individuos primarios y los abstractos o clases, son esquematizables para cualquier marco conforme a la lógica de clases.

(3) Si en nuestras traducciones vertimos «Bello» así, con mayúsculas, del mismo modo que vertimos «Sócrates», queriendo indicar que ambas cosas son individuos existentes - puesto que para Platón las ideas lo son-, pretendemos que nuestro lenguaje sin más enmiendas puede relacionarse con ellas y, con ello, induciendo al lector a posibles errores de interpretación ontológica [23] . Tarde o temprano se podría terminar identificando - no sin razones- a las ideas con términos generales o con clases lógicas reificadas [24]. Pero no parece ser esa la propuesta platónica: Platón no parece identificar las ideas con colecciones (o ausencias =“clases vacías') de entidades, y para nosotros, las clases no tienen valor causal sobre sus miembros; son éstos los que, para nosotros, primariamente hay/existen.

(3.1) Sin aceptar la propuesta platónica en $a$. y puestos a analizarla 'desde fuera', ¿cómo habría que simbolizar lógicamente el enunciado 'Sócrates se relaciona con lo Bello'?. El lector desprevenido, aun cuando estuviera advertido de que 'Bello' alude a una idea - algo que no es de este mundo- y no a un sensoperceptible, podría esperar encontrar la idea de belleza en el mobiliario del mundo o universo al que Sócrates pertenece. El problema no se soluciona agregando un nuevo símbolo (v.g., ' $\Phi$ ' como nombre propio o variable de idea) para este nuevo tipo de entidades, puesto que éstas no son para Platón entidades con el mismo estatuto de realidad que Sócrates. Las ideas no llegan para sumarse a nuestro marco ontológico, como la montaña de oro de Meinong. La invitación del maestro de Aristóteles no es creer en esa ampliaciónontológica. Las ideas son los constituyentes primarios del marco platónico. Así, su invitación es una a cambiar de marco: si la aceptamos, entonces lo sensoperceptible, el mundo fenoménico, queda - por ese universo - consecuentemente subvaluado en la jerarquía ontológica. Y el lenguaje que sea debe dar cuenta del compromiso. Ciertamente, no tenemos razones filosóficas para convertir a su doctrina en una fábrica de posibles destinados a poblar los suburbios de nuestra ontología aceptable [25]: aceptando la de Platón, debemos desestimar la nuestra a la vez que las relaciones con el lenguaje que de ella da cuenta.

(3.2) Así, en el marco platónico no podemos utilizar constantes de individuo $(a, b, c, .$.$) como símbolos de nombres propios (o$ variables de individuos $-x, y, \ldots-$ como sus lugares) en el cálculo lógico que puedan aludir a cosas del tipo de Sócrates. Esto es crucial. Lo que para nosotros eran nombres propios de entidades únicas (individuos), las cuales eran capaces de hacer verdadero el predicado de una descripción definida, han perdido ese estatuto: se han convertido en nombres de grupos de cosas, de agrupaciones de imágenes, cada una de las cuales, por participación a una única y misma idea, puede tener esa relación en común a otras [26]. 
(3.2.1) Las entidad strictu sensu, autê hê ousia (78d1), ahora es la idea, lo restante sus 'imágenes' y éstas ya no pertenecen al universo primario $U$ : recorren otro universo $U 1$ de cosas 'familiares' generalmente vinculadas a las de $U$ por participación o algún otro tipo de relación. ¿Cómo hacer para contar con un lenguaje simbólico adecuado a la ontología platónica conectada a las relaciones 'lógicas' expuestas? En otro lugar mostraré que eso es posible si nos atenemos a 3.2; i.e, si un sensoperceptible es asimilable a un racimo de cualidades del que, en general, cuando hablamos de él, resaltamos una [27]. Para Platón las cosas de $U 1$ tienen un grado de realidad no muy diferente - por no ser primarias - al que tienen las abstracciones, o las clases, para nuestra visión ontológica - cf. (6) infra.

(3.2.2) Dado 3.2 3.2.1, hemos cambiado de marco ontológico. Entonces, aludiendo a su plano primario, podríamos decir que la idea de 'impar' es 'el objeto $y$ tal que $y$ es-impar' (donde 'I'puede reemplaza a 'es-impar' e ' $y$ ' al lugar de una ousía, i.e. de una idea). Lo que podemos simbolizar con $(l y)(I y)$. Con lo cual, si ' $I$ ' es verdadero de más de un individuo de $U$, o de ninguno, (ly)(Iy) no existe. El carácter unitario [28] de las ideas las hace aptas para ser aludidas por las descripciones definidas. Esto nos obliga a pensar que la idea de tres - simbólicamente, $(1 x)(T x)$ - , autê hê ousia que hace verdadero el predicado representado por ' $T$ ', no se identifica con la idea de impar. Pero ello

(3.2.2.1) no impide una relación asimétrica no lógica (o metafísica) entre las mismas (=en $U$ ) que pueda mostrarse o sugerirse por el estudio, justamente propuesto por Platón, en los logoi (i.e. en sus imágenes lingüísticas antes que en las fácticas [29]]), donde 'G' reemplaza al predicado 'genera'.]] =en $U 1$ ) de estas cosas que son (ta onta).

(3.3) Luego, expresiones del tipo 'para todo $x, x$ es $F$ si, y sólo si, $x$ participa de $\Phi$ ' son carentes de sentido tanto en el marco ontológico platónico, como en el nuestro. No nos permiten discernir por el lenguaje cuál es la realidad primaria de la que se está hablando y no concebimos un tercer marco ontológico neutro respecto del cual tal enunciado pueda resultar verdadero o falso. Ese esquema no da cuenta de lo que dice (e2) para Platón, ni para nosotros. No es posible hallar para él ningún ejemplo de sustitución aceptable en los marcos que tratamos: los individuos que 'ocupan' $x$, y $\Phi$, pertenecen a la realidad primaria de marcos ontológicos diferentes - cf. (2) y n.2 infra -, pues las expresiones ' $\mathrm{x}$ ' $\mathrm{y}$ ' $\Phi$ ' deben manifestar compromisos jerárquicos diferentes.

(4) Hemos visto en $b .1$ que las cosas que entrañan a otras son proposiciones, cosas del lenguaje (o nociones susceptibles de ser enunciadas proposicionalmente). Las entidades primarias comúnmente aludidas por $a, b, c$, etc., (i.e., las ideas - para Platón - , o los individuos sensoperceptibles - para nosotros - , aun cuando a las primeras las simbolicemos con $\Phi$, $\Gamma$, etc.) no entrañan ni son entrañadas por nada [이].

(5) Con todo, podría pensarse que mi interpretación de la relación de sumphônein como entrañamiento impide cualquier tipo de jerarquía o asimetría entre las ideas (v.g.de fuego y calor, de tres e impar, etc.), algo que parecen sugerir los ejemplos de Platón sobre la explicación causal 'ingeniosa' (Phaid. 105b8ss.). Ciertamente, podría objetarse que (o) el que algo sea impar no entraña que sea tres.

Como he adelantado, no es este el lugar para explicar cómo los enunciados se entrañan recíprocamente en la táctica platónica dada a los filósofos. Pero, para evitar confusas concepciones, deben tenerse en cuenta los puntos cruciales (1) (3.3) relativos al 'algo' que se pretende mencionar en $(o)$ y al marco ontológico respecto del cual esa proposición puede resultar verdadera o falsa. La conclusión (3.2.2.1, supra) muestra parte de la inmunidad que mi interpretación tiene frente a lo que se concibe al afirmar $(o)$.

(5.1) En segundo lugar debemos descartar que (o) aluda a una asimilación incorrecta de la nuestra a la posición de Gregory Vlastos (1988). En efecto, para la explicación de la aitia 'ingeniosa', él ha propuesto una relación de 'entrañamiento' asimétrica entre las 'Formas' $\Gamma-\Phi$ (v.g. Tres-Impar) que intenta recoger lo que Platón menta conepipherein. La fórmula más elaborada por la que V. explica esta aitia es:

'x es $F$ porque, siendo $G$, ella debe participar en $\Gamma$; y puesto que $\Gamma$ entraña $\Phi$, x debe también participar en $\Phi$, y por lo tanto debe $\operatorname{ser} F^{\prime}[\underline{31}]$.

Es claro que no es de esa relación de la me he ocupado en este trabajo, sino de la que involucra a los logoi que entran en el deuteros plous; huelga decirlo, de sumphônein. Por lo demás, a su formulación 'lógica' le caben las observaciones 33.3 [32], a la vez que presenta aquí una relación de 'entailment' que a su juicio llega a conectar 'concepts' y, como manifiesta su fórmula, son identificados con las 'Formas', esto es, con las ideas ( $c f$. n.30): omitiendo (4) Vlastos parece concebir el entrañamiento entre ideas como una relación semántica semejante a aquella que distingue a los juicios analíticos de Kant [33]. Creo que no hay suficientes razones para asumir esa asimilación que invita a pensar que los sensoperceptibles 'participan' de conceptos o son sus imágenes.

(6) He dicho que los predicados por los que se determinan las entidades deben permitir el entrañamiento de los enunciados (en el caso de la aitia 'ingeniosa', recíprocamente). Pero una entidad es una idea, autê hê ousia (78d1-79a11), no una sensoperceptible. Como propone Platón, la idea de tres 'lleva consigo' (epipherei) la idea de impar. Esto es, cuando esa idea sea un miembro de alguna agrupación sensoperceptible unitaria (como Sócrates, según 3.2 3.2.1), lo será también la otra. El ejemplo de la familia Jones de Vlastos [34] tiene en común con todas las cosas que son ternas el hecho de que es una imagen, una clase del tipo $(1 y)(I y) ;(1 x)(T x) ; \ldots$. Esto fundamentaría la relación de entrañamiento (sumphônein) entre enunciados causales o explicativos (de la aitia 'ingenua' y de 
la 'ingeniosa', v.g.), pero - por (4) - no esuna relación de entrañamiento entre ideas. La conexión entre éstas es metafísica y, aun cuando está supuesta, no es tema central de análisis en los párrafos que Platón dedica al método relativo a los logoi (cf. n.29).

(6.1) Si aceptamos la existencia de las ideas, y decimos que 'algo' es impar, decimos que hay un único individuo (una idea) que es tal. Lo mismo, para el tres. No hay, para Platón, más de una idea de impar, ni más de una idea de tres. Entonces, el 'algo' al que alude la objeción (o), sólo podría ser un solo 'algo': una única entidad $a, b$ o $c$, etc. Luego, por (4), la objeción (o) no tiene sentido al interior de la ontología platónica primaria.

(6.2) Si el 'algo' no es una idea, es un sensoperceptible: algo del mundo fenoménico. La objeción (o) debe decir, por tanto, 'que algo de nuestro mundo fenoménico sea impar, no entraña que ese algo sea tres'. Esto es, que $\varnothing((\mathrm{j} x)(I x P T x))$, donde ' $x$ ' es lugar del fenómeno sensoperceptible que, desde nuestra perspectiva ontológica, corresponde a una entidad primaria (no a una idea) que diferenciamos de sus propiedades simbolizadas en predicados. Esto es, según (2), para afirmar (o) hemos tenido que desestimar la propuesta de Platón en (a.). Así, o bien hemos vuelto a nuestro marco ontológico, o pretendemos hablar de U1. Luego, (o) no dice nada acerca de las ideas según Platón las concibió en su marco, por lo cual no se sigue que, aun en el caso de que $\emptyset$ ((j $x)(I x P$ $T x$ ), las ideas no puedan tener una relación jerárquica entre ellas en $U$.

En fin, la propuesta de Platón es una a ver todas las cosas de otra manera y aceptándola, ya no podemos ver a los sensoperceptibles como individuos capaces de ser el referente de un nombre propio o una constante, pero tampoco a nuestras afirmaciones usuales como ejemplos de sustitución de esquemas o funciones proposicionales de explicaciones causales.

Puesto que hay que cruzar el umbral ontológico de (a.) y aceptar sus relaciones constituyentes, sólo cuando hayamos 'fijado' el estatuto ontológico de lo sensoperceptible en una simbología que lo distinga claramente de lo ideal y contemos, además, con los símbolos lógicos que conecten las cosas más reales de $U$ con las menos de $U 1$ según Platón lo previó, podremos formular las proposiciones (o sus esquemas) del marco ontológico unívoco que deben entrañarse recíprocamente, no antes. Estas proposiciones o aitiai [ 35$]$, explican dentro de su marco ontológico, no fuera de él.

Quedan muchos puntos por precisar, sin dudas, pero nada parece impedir - hasta aquí - la tarea, no menor por cierto, de explicar a Platón en sus propios términos, aun cuando se lo haga desde una perspectiva 'lógica'. [36]

\section{EDUARDO HÉCTOR MOMBELLO}

Centro de Estudios Clásicos y Medievales, Universidad Nacional del Comahue, Neuquén.

Argentina

bibiographie

BEDU-ADDO, J., T. 'The role of hypothetical method in the Phaedo', en Phronesis 24 (1979), 111-32.

Bluck, R., S., 'Hupothesis in the Phaedo and Platonic Dialectic', en Phronesis 2 (1957), 21-31.

Burge, L., E. 'The Ideas as Aitiai in the Phaedo', en Phronesis 16 (1971), 1-13.

EgGers Lan, C., El Fedón de Platón, Buenos Aires, 1993.

Lyons, J. Semántica Lingüística - Una Introducción, tr. Santiago Alcoba de «Linguistic semantics. An Introducction, Cambridge University Press, 1995», Barcelona, 1997.

Quine, W., V., O. Los métodos de la lógica, Barcelona, 19692.

Quine, W., V., ‘Acerca de lo que hay’, en Desde un punto de vista lógico (1984), Buenos Aires, 25-47.

Reale, G., Per una nuova interpretazione di Platone [...] Milano, 199720.

REALE, G., Platón. En búsqueda de la sabiduría secreta, Barcelona, 2001.

Robinson, R., Plato's Earlier Dialectic, Oxford, 19532.

Ross, D., Teoría de las ideas de Platón, Madrid, 19974.

Russell, B., A., W., 'On Denoting', Mind (1905) 14: 479-93; repr. in Logic and Knowledge Essays 1901-1950, ed. R.C. MARSH, Routledge, 1992 (hay traducción española en Valdéz Villanueva, L., ed., -1991- La búsqueda del significado, Madrid, 46-56)

SEDLEY, D. 'Platonic Causes', en Phronesis 43 (1998), Princeton, 114-132.

TAylor, C., C., W., 'Forms as Causes in Phaedo', en Mind 78 (1969), 45-59; repr. en N. SMith, ed., Plato: Critical Assessments, vol. 2, London New York, 1988.

Vlastos, G., 'Reasons and Causes in the Phaedo', en Philosophical Review 78 (1969), 291-325; repr. en N. SMITH, ed., Plato: Critical Assessments, vol. 2, London New York, 1988. 
Footnotes

[1] Así, por ejemplo, Taylor (1988: 3-15) concluye que es imposible descubrir ningún método coherente subyacente a los ejemplos de Platón (cf. p.11). Cf. también la esquematización 'logical or quasi-logical' de Sedley (1998: 115-16); y de otra manera las superaciones nomotéticas afines de Burge (1971: 5), Vlastos (1988: 20 ss.), Taylor (1988: 5-11), et alia. Respecto de la diferencia establecida por el profesor Bluck (1957: 21ss) entre los logoi de 99e5 entendidos como definiciones socráticas, y lashupotheseis de 101d comprendidas como nociones provisionales de las formas, cabe señalar que la distinción no será relevante para el estudio sobre sumphônein que sigue, en la medida en que ambas puedan ser tomadas como proposiciones (o contenidos proposicionales de otro tipo de enunciados) cuyos valores de verdad determinen el de la relación completa ( $c f$. nota 9 infra).

[2] Aun cuando este lenguaje formal en su forma más utilizada - la del uso de funciones proposicionales y cuantificación - no hable de los individuos particulares ontológicamente 'familiares', los supone como aquello único capaz de ser el referente del término por el que se sustituye una constante de individuo en una proposición singular. El que dicha lógica cuente entre sus símbolos fundamentales a aquellos que significan un individuo y su propiedad es condición suficiente para que haya alguien capaz de conocer «la diferencia que hay entre los individuos que tienen propiedades y las propiedades que ellos pueden tener»: Copi, I.

M. Introducción a la lógica, Buenos Aires, 197111, p. 274. Se sigue que de no haber tal conocimiento, no hay lógica, al menos no de este tipo.

[3] Esto es, el recurso de lógica formal a un sistema deductivo consistente cuyos conectivos sean todos extensionales o 'truthfunctional'.

[4] Op. cit. p. 133; cf. también paso (4) en p. 134.

[5] Para la realización de este trabajo he seguido la edición del texto griego de Phaid. editado por Burnet, J. (1900, reed. 1967) en Platonis opera, vol. 1, Oxford. En adelante las citas sin el nombre de diálogo corresponden todas a Phaid. Tal vez no sin razón, Reale (2001: 176) ha argüido que el pasaje que nos ocupa 'presenta la primera demostración de un ser meta-empírico, supra-sensible, y trascendente'. Sin embargo, creo que no se ha logrado captar adecuadamente, desde el punto de vista lógico, en qué consiste tal demostración, si es que la hay: en ibid., v.g., el tratamiento del pasaje que nos ocupa está flagrantemente ausente (cf. op. cit. 175-81). Sin perjuicio de ello, el 'segundo rumbo' ha tenido un amplio tratamiento; cf. v.g. op. cit.; Reale (1997); Burge (1971: 1 n. 2); Robinson (1953: 121-45); Bluck (1957).

[6] Una clara exposición sobre el alcance de esta noción en Phaid. 92c11-d4. Cf. también Tim. 48d2, 53d6, 55d5, 56a1, 57d6, $68 \mathrm{~b} 7$. El carácter provisional que se muestra en estos tres aspectos (a.13) es coincidente con lo que Bedu-Addo (1959: 115) ha señalado como del ámbito de la 'opinión', aunque la propuesta de la acepción de la teoría de las ideas sea aquí mucho más que un mero supuesto.

[7] En aquel pasaje esta hupothesis remite a la exposición de lo que enuncia el logos del que se habla en 75c10-d5.

[8] Cf. $75 \mathrm{~d} 2$. Aunque es irrelevante para el punto, sigo - con un matiz decididamente existencial - la enmienda de Burnet a «touto, ho esti».

[9] Se habla aquí de «contenido proposicional» pues las relaciones lógicas que se involucran en esta exposición comprometen proposiciones y no enunciados. El contenido proposicional es, de modo estándar, aquello que pertenece a un enunciado y puede tomar valor de verdad. Los logoi a los que se alude en los contextos de explicación ontológico-causal suelen ser enunciados no extensionales con contenido proposicional y no pueden, en sentido estricto, ser tomados por proposiciones sin más. Sin embargo, fuera de los contextos estrictamente formales de este trabajo, se seguirá hablando de logoi como de enunciados o proposiciones indiscriminadamente.

[10] Es posible encontrar no menos de ocho matices diversos.

[11] Cf. Crat. 415b2, 420c, 433b4, 436d, 436e.

[12] Nótese que si - a partir de la propuesta de Platón en el pasaje - asumiéramos que lo que «kakia» significa recibe este nombre del significado de lo que «kakos ion» significa, lo significado por el primero sería - según Aristóteles - parónimo de lo significado por el según término (Cf. Categorías 1a12-15).

[13] No hay una simbolización estándar para la relación de entrañamiento. Aquí se utiliza ' $P$ ' como abreviatura de 'entraña'.

[14] No hay acuerdo generalizado sobre la noción de entrañamiento. Por lo general, se acepta que las dos restricciones establecidas para esta relación se vinculan en una conjunción, lo que simbolizamos con 'Ù ': así la utilizaremos. Sin embargo, a la hora de ejemplificar tal relación, algunos lo hacen del siguiente modo: asignan a ' $q$ ' «Aquiles mató a Héctor», y a 's', «Héctor murió», lo cual, si Héctor murió por otras razones que no incluyan la intervención de Aquiles, no cumple con la segunda restricción, a saber, que la falsedad de $s$ se siga necesariamente de la falsedad de $q$. Con tales ejemplos se está pensando, más bien, en una disyunción que en una conjunción. $C f$. Lyons (1997: 143). Podríamos decir que «x es soltero» entraña a «x es no-casado», siempre y cuando restrinjamos todo uso posible de ellos al único que permite que ambos resulten intercambiables en todo mundo posible. Sólo así éste será considerado, en adelante, un buen ejemplo de entrañamiento entre proposiciones.

[15] Platón habla de un enunciado errômenestatos puesto en relación con 'eso' que consuene con él. Intentar establecer a qué se refiere exactamente en este caso no resulta un problema menor, lo que no debe dejarse de tener en cuenta a la hora de medir el alcance de la esquematización que aquí se está proponiendo.

[16] No hay que pasar por alto que el operador 'entraña' no es funcional de verdad (i.e. un conectivo extensional).

[17] Ciertamente, el único aspecto necesario que se ha supuesto hasta el momento es que si un logos concuerda con su logos errômenestatos no puede ser de otro modo que verdadero; y esto, simplemente, porque el texto así lo expresa. 
[18] Se ha sostenido que el enunciado $s$ es tautológico - cf. Eggers Lan (1993: 200 n. 226) y Ross (1997: 51)-, aunque creo que analizando detenidamente el asunto no resulta tan claro: nótese que no hay otro modo de decir que tal enunciado es tautológico si no es susceptible de una esquematización lógica, ya sea perteneciente al marco de nuestra ontología, al de la de Platón, u otra. (Cf. c.2 ss). Coincido con Burge (1971: 6) en que lo que se afirma en esta proposición 'is not a pure tautology: an ontological commitment had been made before the solution was given and is implicit in the schema offered.'

[19] Cf. Russell (1992).

[20] Cf. Quine (1969: 292ss. '§ 36 Descripciones’).

[21] Cf. 74a11-12; 78c3-4; 100b5-9; 102a10-b6; y nota [6] supra.

[22] Noción completamente afín - en lo que a sus derivaciones concierne - a la de 'marco de un determinado esquema conceptual' de Quine (1984: 36).

[23] Estamos obligando al lector a superpoblar su universo, de un modo no muy diverso a aquel que pretendían los señores McX e Y Griega, y del que amargamente se quejaba Quine (1984: 25-38).

[24] $C f$. Quine (1969: 303-4). Cf. n. siguiente.

[25] Esta es una de las razones por la que las críticas de Quine no alcanzan a la propuesta de Platón.

[26] Sin embargo, la interpretación usual no ha advertido el cambio de estatuto por la aceptación del marco ontológico de Platón, toda vez que se ha pretendido utilizar herramienta lógica de nuestro marco sin enmiendas al suyo. Cf. por ejemplo, Vlastos (1988: 20) ítem (2).

[27] De este modo aludimos a un fenómeno de $U 1$ con 'eso es blanco' o 'aquello es tres', aun cuando dicho fenómeno 'refleje' otras cosas reales de $U$.

[28] No es posible demorarnos aquí sobre ese carácter usualmente admitido para las ideas, pero $c f .74 a 9-74 c 5$; 78d1-79a11. De él también suelen dar testimonio las personas de los verbos conjugados relacionados con ellas. Tal el caso de 76d7-9, donde Sócrates pregunta si «existe la $\langle x\rangle$ que (há) siempre repetimos una y otra vez, $x$ que cumple con el requisito de hacer verdadero un enunciado del tipo « $(1 x) F x »$ a la ousia. Ésta es el objeto $x$ que recibe los predicados; cumple en cada caso con esa fórmula; y (78d5-6) es un único aspecto o carácter (monoeides) en sí mismo y por sí mismo. Es importante notar que no está hablando del objeto $x$ tal que $x$ esblanco en sí, o es-bello en sí, etc., sino de cada uno de los xsin más determinaciones. Platón alude a él marcando su falta de carácter o forma en cuanto se habla del lugar del objeto que permanece estable, con auto ho esti (78d3 y 5); lo que podríamos leer como «el objeto $x$ tal que $x$ es -...»; donde los puntos suspensivos corresponden al lugar del carácter-predicado correspondiente a la ousia en cuestión.

[29] No debemos olvidar que, como advierte Sócrates en 100a ss., esto es un examen en imágenes (eikones) de las cosas que son, tanto como lo es el que se realiza en los hechos (ta erga) - aunque probablemente la copia sea más fiel -. Por otro parte, respecto de la asimetría entre ideas en $U$, las descripciones que forman parte de la hipótesis y de los enunciados entrañados, en sí mismas, no impiden que nuestra interpretación pueda volar a grandes alturas, si se quiere. ¿Quiere recogerse simbólicamente que, en virtud de su morphê, el eidos de tres es impar y no al revés ( $c f .103 \mathrm{e} 2-5 ; 104 \mathrm{a} 7-\mathrm{b})$ ? Entonces puede hablarse del objeto $I(1 x)(T x)$, lo cual no presenta diferencias sustanciales a pensar que la forma de perro es cuadrúpeda. Los predicados no pertenecen a $U$; y las relaciones entre ideas son mucho más profundas de lo que el estudio en los logoi propuesto por Platón alcanza a mostrar para ellas ( $v . g$., que muchas de ellas llevan consigo - epipherei- a otras), ante la necesidad de aludir a las conexiones entre $U$ y $U 1$ (cf. punto 6) para explicar las cosas de este último plano. Con todo, sobre la base de que los contrarios en sí no se generan recíprocamente (103c), se podría querer probar que la idea de impar genera a la del tres y, consecuentemente, aludir a ella. En tal caso, habría que hablar del objeto $(1 y)(I y \grave{U}[(\$ w)[G y w \grave{U}(x)(T x \ll x=w)$

[30] En nuestro marco esto sería un tipo de error categorial, tal como pensar que Sócrates entraña a Cármides: sus estatutos ontológicos no alcanzan al plano en el que tales relaciones tienen aplicación. Por lo demás, no hay razón para pensar que esa relación pueda tener sentido en el marco de las realidades primarias de Platón: el que las ideas sean inteligibles no las identifica con conceptos, tal como no identificamos el estatuto ontológico de Sócrates con el de un concepto por el hecho de que éste pudiera resultarnos inteligible, aun cuando de ambos tipos de entidades primarias pueda darse un enunciado explicativo. Por ello, las entidades primarias tampoco se identifican conconceptos que expresen condiciones necesarias y suficientes para que 'algo' pueda ser llamado de cierto modo

[31] Cf. Vlastos (1988: 27).

[32] Aun cuando $\Gamma$, $\Phi$, etc. representen para él variables de Forma (p.20)-concepto(p.27). Asunción que parece innecesaria dado que la cuantificación de ideas no entra en su formulación.

[33] Cf. Vlastos (1988: 27 y n. 68).

[34] Cf. Vlastos (1988: 28).

[35] En el sentido, que bien ha señalado Burge (1971: 2-3), de explicaciones o respuestas a la pregunta '¿por qué?'.

[36] Estoy en deuda con el Prof. Julio A. Castello Dubra por las discusiones de las que, en buena medida, surge este trabajo.

Agradezco, también, al Prof. Marcelo D. Boeri sus agudas sugerencias; a las profesoras Cristina Behnisch y Verónica Viñao la discusión de algunos tópicos lógicos específicos; las penetrantes observaciones de los evaluadores anónimos de la International Plato Society, y las sugerencias editoriales del Prof. Christopher Gill. 